\title{
Conditionally Active Biologic Anti-AXL Antibody-drug Conjugate BA3011
}

National Cancer Institute

\section{Source}

National Cancer Institute. Conditionally Active Biologic Anti-AXL Antibody-drug Conjugate

BA3011. NCl Thesaurus. Code C156141.

An antibody-drug conjug ate (ADC) composed of a conditionally active biologic (CAB) antibody against $A X L$ receptor tyrosine kinase ( $A X L ;$ UFO) conjug ated to an as of yet undisclosed cytotoxic agent, with potential antineoplastic activity. Upon administration of CAB-AXL-ADC BA3011, the anti-AXL antibody becomes activated through an as of yet not fully elucidated process only under the unique microphysical conditions that are present in the tumor microenvironment (TME) as a result of the glycolytic metabolism of cancer cells, and not in the microenvironment of normal, healthy tissues. Upon selective binding to AXL-expressing tumor cells and internalization, the cytotoxic agent kills the tumor cells through an as of yet undisclosed mechanism of action (MoA). AXL, a member of the TAM (TYRO3, AXL and MER) family of receptor tyrosine kinases normally expressed on many normal, healthy cells and overexpressed by many tumor cell types, plays a key role in tumor cell proliferation, survival, invasion and metastasis; its expression is associated with drug resistance and poor prognosis. The CAB antibody allows for efficient and reversible binding to AXL-expressing tumor cells under conditions that are present only in the TME, thereby maximizing efficacy while minimizing toxicity by avoiding activation and thus binding of the antibody to normal, healthy AXL-expressing cells under normal conditions. 